\title{
A Rare Case of Tumor Lysis Syndrome
}

\author{
Anne Mainardi, MSIV, Daniel Okamoto, MD, Jianqing Lin, MD
}

\section{INTRODUCTION}

Tumor lysis syndrome (TLS) is a metabolic disturbance caused by the destruction of rapidly dividing cancer cells following administration of cytotoxic chemotherapy. The subsequent release of intracellular material results in hyperuricemia, hyperkalemia, hyperphosphatemia, and hypocalcemia. ${ }^{1}$ The clinical presentation of TLS, including acute kidney injury, results from these electrolyte abnormalities and can be life-threatening. ${ }^{2}$ Here we present the second reported case of TLS in a woman with endometrial cancer.

\section{CASE PRESENTATION}

A 63 year old woman with newly-diagnosed endometrial cancer (International Federation of Gynecology and Obstetrics, FIGO, stage IVB) who received her first dose of carboplatin and paclitaxel four days earlier presented to the emergency room with shortness of breath and lower extremity swelling. Her physical examination was significant for a heart rate of 132 beats per minute and a respiratory rate of 26 breaths per minute. She was noted to have a harsh systolic murmur loudest at the right second intercostal space and a mildly distended abdomen. Chest radiography was unremarkable. A ventilation perfusion scan was negative for pulmonary embolism. Her labs at time of admission (Table 1) were consistent with tumor lysis syndrome. Based on the Cairo-Bishop criteria (described below), the diagnosis of tumor lysis syndrome was made.

\section{OUTCOME AND FOLLOWUP}

The patient received vigorous intravenous hydration with normal saline solution and treatment with intravenous rasburicase. After two doses of rasburicase her labs began to normalize (Table 1), and her symptoms dissipated. On the day of discharge all lab abnormalities had resolved, and the patient was discharged in stable condition.

\section{Table 1. Laboratory Values}

Lab Parameter
(normal range at TJU)

Serum potassium
(3.5-5.0 mmol/L)

BUN

(7-27 mg/dL)

Serum creatinine

(0.7-1.4 mg/dL)

Serum phosphate

(2.4-4.5 mg/dL)

Serum calcium

(8.5-10.5 mg/dL)

Serum Urate

(2.5-6.0 mg/dL)

*values were not measured

4.4

14

0.8

$n / a^{*}$

$n / a^{*}$

$n / a^{*}$

5.5

68

2.4

6.1

8.3

15

\section{5}

65

1.9

4.3

8.3

5.1 


\section{Table 2. Clinical Characteristics of Patients at High Risk for Tumor Lysis Syndrome}

Tumors with a high proliferation rate and sensitivity to cytotoxic agents

Large tumor masses

Renal insufficiency and obstructive uropathy

Elevated serum lactate dehydrogenase or uric acid level

Dehydration

\section{TABLE 3. Cairo-Bishop definition of laboratory tumor lysis syndrome in adults.}

Diagnosis requires two or more of the following abnormalities observed within three days before to seven days after initiation of chemotherapy:

\begin{tabular}{|c|c|}
\hline Uric acid & Greater than or equal to $8.00 \mathrm{mg} / \mathrm{dL}$ or $25 \%$ increase from baseline \\
\hline otassium & Greater than or equal to $6.00 \mathrm{mmol} / \mathrm{L}$ or $25 \%$ increase from baseline \\
\hline hosphorous & Greater than or equal to $4.5 \mathrm{mg} / \mathrm{dL}$ or $25 \%$ increase from baseline \\
\hline & ess than or equal to $7.0 \mathrm{mg} / \mathrm{dL}$ or $25 \%$ decrease from baseline \\
\hline
\end{tabular}

Table 4. Cairo-Bishop definition of clinical tumor lysis syndrome in adults.

Diagnosis requires meeting criteria of laboratory tumor lysis syndrome plus one or more of the following not directly or probably attributable to a therapeutic agent:

1 Creatinine greater than 1.5 times upper limit of normal (our ULN $=1.4 \mathrm{mg} / \mathrm{dL}$ )

2 Cardiac arrhythmia/sudden death

3 Seizure

\section{DISCUSSION}

TLS is a rare but serious complication of cytotoxic chemotherapy. Malignancies with the highest risk of TLS are those with both high proliferative rates and tumor burden, particularly hematologic malignancies. ${ }^{3}$ Solid tumors have a much lower incidence of TLS. A literature review found the highest incidence of TLS in small-cell carcinoma and breast carcinoma, with a few reported cases each in neuroblastoma, germ cell tumors, melanoma, and others. ${ }^{4}$ While a few cases of TLS have been reported in association with gynecologic cancers, ${ }^{5,6}$ there has been only one reported case associated with endometrial cancer. In this case from 2010, a 60 year old woman with recurrent FIGO stage IIB endometrial cancer developed TLS four days after receiving carboplatin and paclitaxel. The patient required hemodialysis and expired despite aggressive management?

While TLS remains a rare complication of chemotherapy in patients with solid tumors, the clinical characteristics of patients at high risk for TLS should be recognized (Table 2). ${ }^{8}$ In 2004, Cairo and Bishop proposed a system for diagnosing and classifying tumor lysis syndrome. 
Table 3 describes the laboratory definition of TLS, and Table 4 describes the clinical definition of TLS. Acute kidney injury in TLS results from the release of nucleic acids from lysed tumor cells, which are degraded by xanthine oxidase to hypoxanthine, xanthine, and uric acid. ${ }^{9}$ In the acidic environment that often occurs as a result of volume depletion, uric acid solubility decrease ${ }^{10}$ and crystallization occurs in the distal tubules and collecting ducts. This crystallization obstructs the tubular lumen and leads to inflammation. ${ }^{11}$ Uric acid may also contribute to acute kidney injury by crystalindependent mechanisms, due to its vasoconstrictive, anti-angiogenic, pro-inflammatory, and pro-oxidative properties..$^{12}$ The other features of clinical tumor lysis syndrome - tetany, cardiac arrhythmias, and seizures also result from the metabolic derangements that occur when cancer cells are lysed by cytotoxic chemotherapy. ${ }^{13}$ The patient described here did not exhibit these manifestations.

The treatment of tumor lysis syndrome is mainly supportive and includes cardiac and electrolyte monitoring, correction of electrolyte abnormalities, intravenous fluids (isotonic saline solution at 2500-3000 $\mathrm{mL} / \mathrm{m}^{2} / 24$ hours), and renal replacement therapy if indicated. ${ }^{14}$ Urinary alkalinization is controversial. The patient described here received four liters of normal saline and two doses of rasburicase. Rasburicase decreases uric acid levels by catalyzing the conversion of uric acid to allantoin, which is more soluble in urine. ${ }^{15}$ This is in contrast to allopurinol, a prophylactic medication, which competitively blocks xanthine oxidase, preventing the conversion of purines to uric acid. ${ }^{16.17}$ Once TLS is diagnosed, rasburicase is the appropriate therapy to eliminate the excess uric acid. ${ }^{13}$

\section{KEY POINTS}

Successful management and treatment of TLS is highly dependent on the prompt identification of clinical and laboratory characteristics, signs, and symptoms of patients at risk. The initiation of prophylactic measures, especially hydration and administration of allopurinol, and the early recognition and treatment of metabolic abnormalities using rasburicase can prevent the severe and life-threatening complications associated with TLS.

\section{REFERENCES}

1. Cairo, M. S. and Bishop, M. Tumour lysis syndrome: new therapeutic strategies and classification. Brit J of Haem 2004; 127: 3-11.

2. Haas $M$ et al. The spectrum of acute renal failure in tumor lysis syndrome. Nephrol Dial Transplant. 1999; 14 (3): 776-779

3. Hochberg J, Cairo MS. Tumor lysis syndrome: current perspective. Haem. 2008; 93(1): 9-13.

4. Baedsgaard L, Sorensen JB. Acute tumor lysis syndrome in solid tumors-a case report and review of the literature. Cancer Chemotherapy and Pharmacology. 2003; 51( 3): 187-192.

5. Chan JK, Lin SS, MCMeekin DS, Berman ML. Patients with malignancy requiring urgent therapy: Case 3. Tumore lysis syndrome associated with chemotherapy in ovarian cancer. J Clin Oncol 2005; 23(7): 6794-6795

6. Yahata T, Nishikawa N, Aoki Y, Tanaka K. Tumor lysis syndrome associated with weekly paclitaxel treatment in a case with ovarian cancer. Gynecol Oncol. 2006; 103(2): 752-754.

7. Godoy H, Kesterson JP, Lele, S. Tumor lysis syndrome associated with carboplatin and paclitaxel in a woman with recurrent endometrial cancer. Int J of Gyn and Obst. 2010; 109(3): 254.

8. Bishop MR, Cairo MS, Coccia PF. Tumor Lysis Syndrome in Clinical Oncology. Edited by Abeloff MD, Elsevier Inc 2004 (3rd edition) 987-992

9. Gutfreund H, Sturtevant JM. Steps in the oxidation of xanthine to uric acid catalyzed by milk xanthine oxidase. Biochem J 1959; 73(1): 1-6

10. Kanbara A, Hakoda M, Seyama I. Urine alkalinization facilitates uric acid excretion. Nutr J. 2010; 9:45.

11. Kjellstrand CM, Cambell DC, von Hartitzsch B, Buselmeier TJ. Hyperuricemic acute renal failure. Arch Intern Med. 1974; 133 349-59.

12. Shimada M, Johnson R, May WS, et al. A novel role for uric acid in acute kidney injury associated with tumour lysis syndrome. Nephrol Dial Transplant. 2009; 24 (10): 2960-2964.

13. Howard SC, Jones DP, Pui C. The tumor lysis syndrome. N Engl J Med. 2011; 364: 1844-1854

14. Coiffier B, Altman A, Pui CH, et al. Guidelines for the management of pediatric and adult tumor lysis syndrome: an evidence-based review. J Clin Oncol. 2008; 26: 2767

15. Kennedy LD, Koontz S, Rao K. Emerging role of rasburicase in the management of increased plasma uric acid levels in patients with hematologic malignancies. J Blood Med. 2011; 2: 1-6.

16. Krakoff $I H$, Balis ME. Allopurinol in the prevention of hyperuricaemia secondary to the treatment of neoplastic diseases with alkylating agents, adrenal steroids, and radiation therapy. Ann Rheum Dis. 1966; 25(6): 651-654

17. Hitchings $\mathrm{GH}$. Effects of allopurinol in relation to purine biosynthesis. Ann Rheum Dis. 1966; 25(6): 601-607. 